\title{
Control-Relevant Modeling of Hypersonic Vehicles
}

\author{
J. M. Vogel*, A. G. Kelkar*, G. Inger*, C. Whitmer*, A. Sidlinger*, and A. Rodriguez ${ }^{\dagger}$
}

\begin{abstract}
Scramjet powered hypersonic vehicles represent the next critical step toward achieving NASA's vision for Highly Reliable Reusable Launch Systems (HRRLS), affordable space access, planetary re-entry systems, and global reach vehicles. The design of such vehicles is a very interdisciplinary and highly complex problem. As such, the development of varying fidelity mathematical models for assessing overall stability and performance during the design process is very important. In particular, developing "low-order" models with "sufficient fidelity" to capture control-centric phenomena becomes vital in early stages of vehicle design. Historically, the early stage vehicle design process never incorporated control related considerations. The design obtained by such practice is not optimal and can often lead to poor design from a stability and performance view point. This paper presents control-relevant modeling efforts which will facilitate quick iterative control analysis and design during early stages of vehicle design. The paper is intended to be of an introductory nature and presents the high level modeling framework and associated challenges. An example linear 6 DOF model with some representative analysis is also given to demonstrate the applicability of the tool suite.
\end{abstract}

\section{INTRODUCTION}

Hypersonic transportation systems are envisioned as a potential solution for affordable space access[1]. NASA has undergone over 60 years of research on air-breathing hypersonic technology since the development of the hydrocarbonfueled ramjet concept. Early works in this area began in 1960s and continued through the 1990s into 21st century. Despite the significant work done in this area there remain critical technological gaps that need to be filled before a viable hypersonic transport systems can be a reality. It is necessary to advance the capabilities not only in the discipline-specific areas such as materials and propulsion but also in the area of multidisciplinary modeling, analysis, and design to fully understand and model the complex dynamic behavior of these vehicles.

The goal of the recent Hypersonic Project of NASA [1] is to conduct long-term, cutting-edge research in the core competencies of the hypersonic regime, thereby producing knowledge, data, capabilities, and design tools at the foundational, discipline, multidiscipline, and systems levels. These research outputs will address the technical challenges for two high-payoff NASA-unique missions: Highly Reliable Reusable Launch Systems (HRRLS) and High Mass Mars Entry Systems (HMMES). One of the most important capabilities needed to support the NASA's Hypersonic Project is the tool suite that can enable modeling, simulation, analysis,

\footnotetext{
* VSI Aerospace, Inc., 2214 229th Place Suite 110, Ames, IA 50014, USA. vsiaero@gmail.com

$\dagger$ Control3D, Tempe, AZ
}

and synthesis of candidate vehicle designs. Such capability has to span across all disciplines due to multidisciplinary nature of hypersonic vehicle dynamics. This paper presents some of the on-going work in the area of control-centric 6 DOF modeling of hypersonic vehicles. Some early results in the this modeling tool suite development are also given as appropriate.

\section{RELATED WORK AND MOTIVATION}

Modeling of hypersonic vehicles is a very challenging problem due to significant interactions between aerodynamics, propulsion, structures, and control. There exist a large body of literature on the modeling of such vehicles and associated challenges, for example see [2], [3], [4] , [5], [6], [7], [8], and [9].

The first attempt at a comprehensive analytical model of the longitudinal dynamics of hypersonic vehicle was undertaken by Chavez and Schmidt [3] wherein Newtonian Impact theory was used to model flow field dynamics which allowed a rather straightforward determination of the pressures acting on the vehicle. The pressures were dependent upon vehicle Mach number, freestream pressure, angle-of-attack, and the vehicle geometry. The equations for total aerodynamic forces were then a linearized to give analytical expressions for the stability and control derivatives. Some control synthesis was also given for the linearized model. In Rudd and Pines [10], a CFD code was used to study the propulsion/airframe integration and determination of the stability and control derivatives. These derivatives were compared to those estimated using hypersonic piston theory. The vehicle design included a translating engine cowl to maintain a shock-on-lip condition. It was discovered that the derivatives had inaccuracies caused due to inapplicability of hypersonic piston theory to the engine flow since the Mach numbers within the engine are not hypersonic.

The model presented in Bolender et al [4] captures the structural, aerodynamic, and propulsion system coupling inherent in a scramjet powered vehicle. The approach taken here relaxes some of assumptions of the model given in [3]. The two additional effects considered in [4] that were not considered in the references cited above included the on-design and off-design propulsion system performance resulting from an oscillating bow shock and the presence of coupling between the rigid body accelerations and flexible body dynamics. The effects of inlet mass flow spillage due to an oscillating bow shock was captured using an oblique shock and Prandtl-Meyer expansion theory rather than Newtonian impact theory. The flexibility of fuselage was captured by modeling the vehicle as two cantilever beams clamped at 
the center-of-mass of the fuselage. The centrally clamped cantilever beams do not give realistic mode shapes. This drawback was later corrected in [9] by modeling the airframe as a free-free beam. In recent literature [8]-[9] the fidelity of models developed earlier was extended to include improved propulsion model which accounts for pre-combustor shock train, fuel mixing and finite-rate chemistry. There are several other papers which are also worthy of citation but could not be included here due to space constraints.

In summary, there exists an extensive literature in discipline-specific areas that discuss modeling of various aspects of scramjet-powered hypersonic vehicles. Most of these pioneering efforts, while multidisciplinary and physics based, do not readily lend themselves to help develop control-relevant dynamic models having higher order with desirable fidelity. The work focused on deriving higher fidelity computational models using high power CFD tools and finite element methods suffers from a drawback that it requires large amounts of time to spin off a fully integrated control design model which is particularly not desirable in the early stages of the vehicle design.

This paper presents a modeling framework that is designed to obtain nonlinear and linear 6 DOF models having varying degrees of fidelity using approximate but efficient engineering methods. This is in contrast to the most of the above mentioned literature which primarily focused on developing 3 DOF model. Moreover, the emphasis has been on developing models with decoupled longitudinal and lateral dynamics. The tool suite proposed in this paper aims at developing models with fully coupled longitudinal-lateral dynamics to improve fidelity by capturing the effects of coupled dynamics. For example, non-zero sideslip can affect pressure distribution on the forebody, mass flow at the engine inlet leading to change in engine performance at the inlet and exhaust, and associated control requirements. The example vehicle configuration modeled to demonstrate the application of modeling tools described in this paper is NASA's Vision Vehicle (an example of HRRLS class of vehicles).

\section{PROCESS Flow AND ASSOCIATED MODUlES}

The guiding philosophy behind the modeling framework presented here is to develop a suite of multidisciplinary modeling tools which integrate disciplinary states-of-the-art to enable timely development of dynamic models of concept vehicles with varying fidelity and plant size. These tools will facilitate control-relevant analysis and design at early stages of the vehicle design process to maximize overall vehicle stability and performance. The idea is to develop models with required fidelity to answer key questions such as what are the stability properties of the design in question; which parameters impact stability margins the most and by what amount; how much structural flexibility can be tolerated; what is the controller bandwidth and is it sufficient to meet missions control objectives; etc. The critical aspect in the model development is to balance the fidelity with the computational efficiency.
The high level process flow used in our model development is given in Fig. 1. The figures shows four main process modules - user inputs, main processing, model extraction, and postprocessing. Each of these modules contain one or more sub-modules dedicated to a specific aspect of modeling process. Given below is brief description of the key modules:

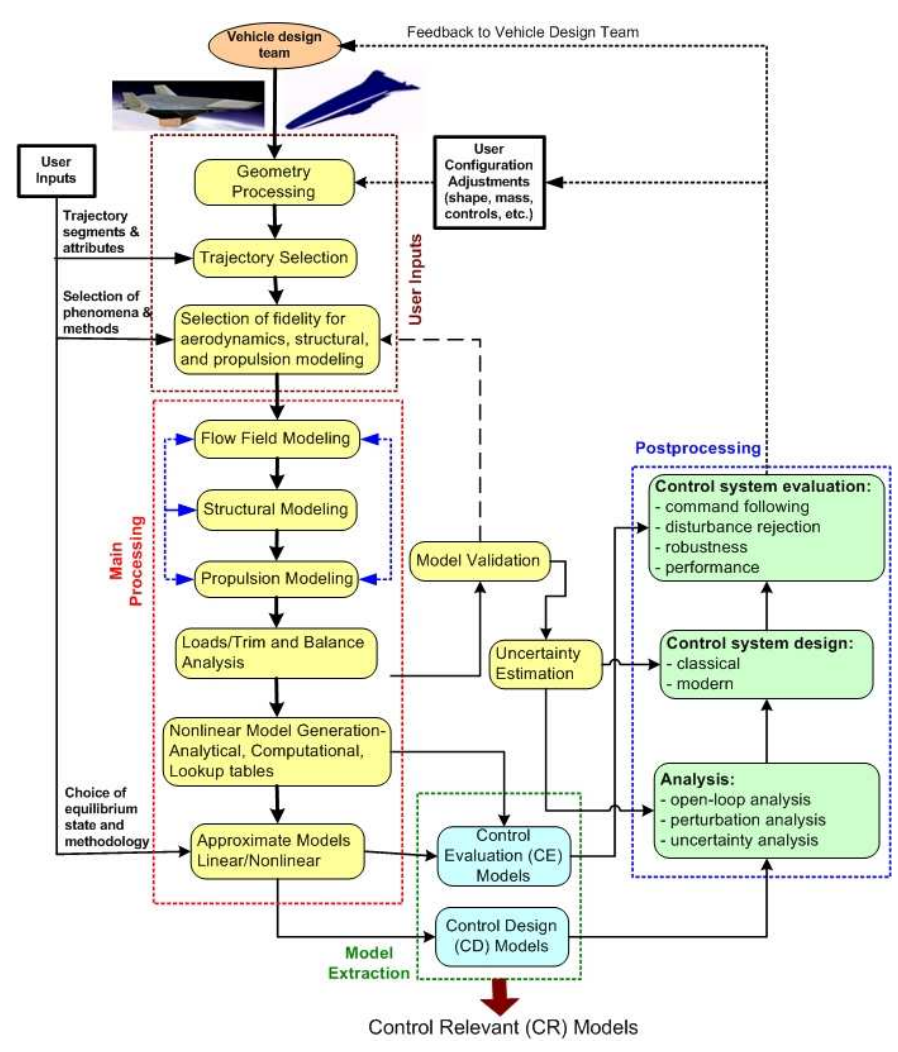

Fig. 1. Control-Relevant Modeling Environment Functionality Flow Diagram

A. User Input Module

This module is intended for various types of user inputs. It contains various submodules which essentially function as pre-processing units before various analysis are performed. Through GUI interface user can select various options ranging from geometry, type of analysis, to level of fidelity needed. Some key sub-modules contained in this module are described below.

1) Geometry Processing Module: This module has capability to import and pre-process concept vehicle geometry, mass distribution definition, and internal structure/stiffness definition databases. The primary function of this module is to develop the surface grid with $\mathrm{n}$-regular regions (panelling) to a desirable level of resolution in such a way that it facilitates computation of aero-propulsion-structure dynamics in seamless manner. The module is also designed to have capability for generating small perturbations in baseline configuration to conduct sensitivity studies to analyze the impact of perturbations on control system stability and performance. This module has user friendly GUI to facilitate efficient analysis. Figure 2(B) shows perturbation in the overall size of the vehicle along its longitudinal axis. 
Once the panelled geometry is available the force and moment computation codes from main processing unit can be used to determine the forces and moments on the vehicle for any given flight condition. The overall forces and moments are simply the summation of forces and moments acting on each panel. For example,

$$
F_{x}=\sum_{i=1}^{N_{\text {pan }}} F_{x i}, F_{y}=\sum_{i=1}^{N_{\text {pan }}} F_{y i}, F_{z}=\sum_{i=1}^{N_{\text {pan }}} F_{z i}
$$

where $N_{\text {pan }}$ is the total number of panels, $F_{k i}$ is the force acting in the $k$ direction at the centroid of the $i$-th panel, and $\mathcal{L}_{i}, \mathcal{M}_{i}, \mathcal{N}_{i}$ are the roll, pitch, and yaw moments acting on the $i$-th panel.

$$
\mathcal{L}=\sum_{i=1}^{N_{\text {pan }}} \mathcal{L}_{i}, \mathcal{M}=\sum_{i=1}^{N_{\text {pan }}} \mathcal{M}_{i}, \mathcal{N}=\sum_{i=1}^{N_{\text {pan }}} \mathcal{N}_{i}
$$

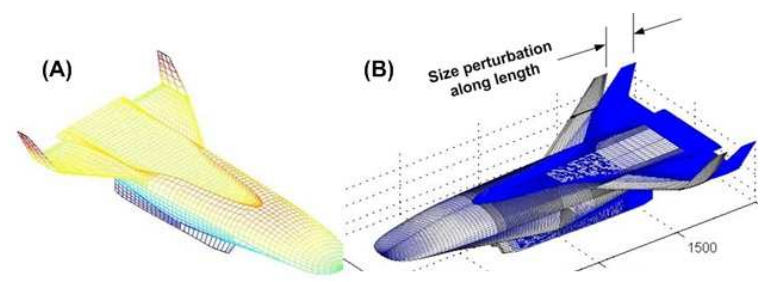

Fig. 2. Vision Vehicle with panelled geometry and length perturbation

2) Trajectory Processing Module: This module is not intended for synthesizing the trajectory but to process the trajectory information obtained from mission requirements. The main functionality includes selection of trajectory components, concatenation of different trajectory sections to obtain an overall trajectory, and determination of associated vehicle dynamic states at selected trajectory points.

\section{B. Main Processing Module}

1) Flow Field Analysis Module: The primary function of this module is to compute aerodynamic pressure and shear force distributions over the vehicle surface for a given flight condition. It is designed to contain several sub-modules representing different flow code algorithms having different levels of fidelity and computational efficiency.

The primary inviscid flow field module currently available incorporates Van Dyke's Unified Super/Hypersonic Similitude Principle that doubly-extends the applicability of the well-known Tangent Wedge concept to include both the strongly hypersonic Newtonian-like limit while also embracing the lower small disturbance supersonic flow limit. A primary concern in this development is those many local regions where the local flow is both supersonic (but not necessarily hypersonic) and only moderately inclined with respect to the freestream. The method yields a pressure field given by

$$
\begin{aligned}
C_{p L} & \simeq 2 \sin ^{2} \theta_{L}\left[\sqrt{\left(\frac{\gamma+1}{4}\right)^{2}+\left[\left(M_{L}^{2}-1\right) \sin ^{2} \theta_{L}\right]^{-1}}\right. \\
& \left.+\left(\frac{\gamma+1}{4}\right)\right]
\end{aligned}
$$

This modified theory also provides a companion relationship for treating leeward panel regions that eliminate the need for shock expansion theory in the aerodynamic calculations. This new model provides an easily used yet still relatively simple analytical pressure relationship that significantly improves on the simple Newtonian Theory and can be used over a much wider range of local Mach numbers on the windward side of the vehicle. The method is more analytically tractable in that it eliminates the cumbersome panel-to-panel application along the body. Pressure prediction on local panels that lie on the leeward surface of the vehicle based on the proposed modeling is given by

$$
\frac{C_{p_{2}}}{C_{p_{1}}} \simeq\left[1-\left(\frac{\gamma-1}{2}\right) \frac{M_{1}^{2} \Delta \theta_{2}}{\sqrt{M_{1}^{2}-1}}\right]^{\frac{2 \gamma}{\gamma-1}}
$$

where, $\Delta \theta_{2}$, is the local surface turning angle. Derivation of this function is based on the observation that leeward region flow is typically rather a high Mach number/small turning angle situation. Additional considerations were made of hypersonic Prandtl-Meyer Expansion Theory combined with the Van Dyke Unified Similitude Principle.

Boundary Layer Reconstruction to Capture Viscous Effects:

The reconstruction of the boundary layer in this work is focused on the important regions of the vehicle where the flow is unseparated and involves only weak lateral pressure gradients (small cross flow effects). For the highly-cooled surface flight conditions of primary interest, we employ for this purpose an adaptation of Cheng's local flat plate similarity concept tied to the local inviscid flow streamline pattern on the body. This provides analytical relationship on each surface for the local skin friction, heat transfer, and boundary layer displacement thickness on said surface.

Let $x$ denote the arc length along a chosen streamline to a particular module with the subscript $e$ denoting the inviscid flow properties there. Then the aforementioned theoretical model gives the local skin friction $C_{f}(x)$ on the module and the corresponding heat transfer coefficient $C_{h}(x)$ as:

$$
C_{f}(x) \simeq C_{1} \sqrt{\frac{C_{w}}{F_{p}(x) R e_{x}}} ; \quad C_{h}(x) \simeq \frac{C_{f}(x)}{2}
$$

where $C_{1}$ is a numerical constant of order unity, $C_{w} \equiv$ $\frac{\mu_{w} T_{e}}{\mu_{e} T_{w}}$ is the Chapman-Rubasin viscosity factor, $R e_{x} \equiv \frac{\rho_{e} V_{e} x}{\mu_{e}}$ is the local Reynolds number and

$$
F_{p} \equiv \frac{1}{\left(\frac{p_{e}(x)}{p_{\infty}}\right)^{2} x} \int_{0}^{x} \frac{p_{e}(x)}{p_{\infty}} d x
$$

is a pressure distribution function evaluated from the known pressure field along the various panels. The corresponding displacement thickness is given by the analytical expression

$$
\frac{\delta^{*}(x)}{x} \simeq\left(C_{2}+C_{3} M_{e}^{2}\right) \sqrt{\frac{C_{w} F_{p}(x)}{R e_{x}}}
$$


where $C_{2}$ and $C_{3}$ are two other numerical constants of order unity. Although visous-inviscid effects are ignored in our early works, they will be included later - for weak interactions at least - using the above result. Please note that once the local skin friction $C_{f}(x)$ on the module and the corresponding heat transfer coefficient $C_{h}(x)$ is known from the relation (Eq. 5) determination of the temperature field can follow using well known relationships in the literature (for example, see [3]).

2) Structural Analysis Module: Structural analysis module is aimed at modeling flexible dynamics of the vehicle structure. The elastic deformations are assumed to be in the linear range. The model is assumed to undergo both static/quasistatic deformation and dynamic/harmonicly excited deformation. The model is parameterized to allow geometry stretches, loft changes, wing and control surface resizing. The parametrization and ability to perturb structural definition facilitates trade studies both in the aerodynamics analysis and the structural analysis.

The structural flexibility in the model has been modeled with two different levels of fidelity. The simplest one uses analytical beam approximation where the structure of the vehicle is modeled as an equivalent annular beam along the longitudinal $(x)$ axis of the craft. The next higher fidelity model is obtained based on finite element model of the structure. This model is constructed using the assumed modes method. The number of modes to be included in the model are selected based on the fidelity desired and analysis of interest. The reduced order approximations are supplemented by full FEA solutions for determining static deformation when necessary. Temperature effects on modal properties, and static deformations may be considered in FEA analysis.

A linearized elastic dynamics of the structure can be given by the following linear state space equations:

$\ddot{\eta}+D \dot{\eta}+\Lambda \eta=\Xi^{T} \Pi_{\tau} \tau ; \quad D=\operatorname{Diag}\left[2 \zeta_{i} \omega_{i}\right] ; \Lambda=\operatorname{Diag}\left[\omega_{i}^{2}\right]$

In this formulation $\eta$ is the generalized modal coordinate, $\Xi$ is the discrete mass normalized modeshape vector evaluated at corresponding generalized force input locations, and $\Pi_{\tau} \tau$ are the mapped inputs to the structural dynamic equations. A finite element approach also allows the inclusion of thermal loads and structural prestress into the solution process in a "loosely coupled" manner.

3) Propulsion Module: The initial version of our propulsion model will extend the 1-D engine model used in [3] and [4] to include the effects of lateral vehicle motion. The 1-D model consists of an inlet, an isentropic diffuser, a 1-D Rayleigh flow combustor (frictionless duct with heat addition), and an isentropic internal nozzle. The lateral motion of the vehicle causes asymmetric flow condition at the inlet of the engine. Our model incorporates an integrated average of asymmetric inlet condition across the inlet. This will account for the inlet spillage. Recently published paper [9] gives potential avenues to include higher fidelity propulsion dynamics in control design models.

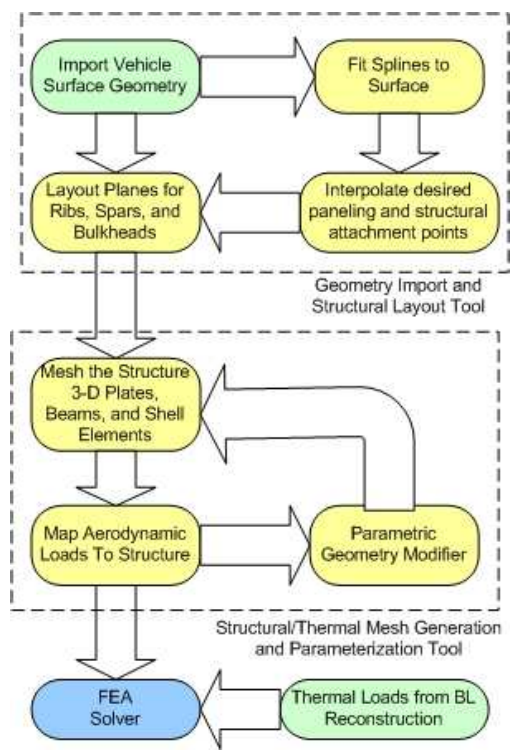

Fig. 3. Interaction between aero-structural analysis tools

Coupling Effects: As stated previously, due to significant interactions between aerodynamics, elastic structure, and propulsion, inclusion of coupling dynamics between these becomes imperative to obtain a meaningful control design and/or evaluation model. The aero-thermo-elastic coupling can be modeled with varying degrees of accuracy. At the simplest level, our modeling process includes "loosely coupled" dynamics. The loosely coupled dynamics uses mode shapes and mode frequencies for undeformed vehicle configuration in elastic dynamics without considering dynamic changes in these modal properties due to temperature changes. However, the temperature effects are considered in determining static deformation. The information flow between geometry processing tools and aero-thermo-elastic analysis tools is depicted in Fig. 3.

\section{Model Extraction Module}

This module's primary function is to extract nonlinear or linear models. The types of the model that can be extracted include: Nonlinear Analytical, Linear Analytical, Nonlinear Hybrid, Linear Hybrid, Nonlinear Computational, and Linear Computational. Analytical models will be generated for a limited number of panels using symbolic solvers. These models will allow user to see closed-form functional relationship between different parameters of the system. The trade-off is the fidelity of these models will be inherently limited. Purely computational models will consist of databases of forces and moments for a series of flight conditions on a given trajectory. If desired, the hybrid models can also be generated wherein the stability derivative terms in nonlinear or linear models are determined using embedded computational code. The nonlinear analytical models can also be based on closedform curvefit functions obtained from force and moment databases or stability derivative databases.

\section{Post Processing Module}

The post processing module consists of open-loop and closed-loop controls analysis module, control synthesis mod- 
ule, and uncertainty analysis module. Control analysis module allows various control-centric analysis such as frequency response analysis, pole-zero analysis, non-minimum phase behavior, inherent performance limitations, etc. The control synthesis module contains different templates for designing either classical or modern control designs.

1) Control Analysis and Design Module: Control Analysis module is designed to conduct parametric equilibrium studies such as, parametric root loci, static margin analysis, control surface sizing/location/segmentation analysis for trim states, and parameter-dependent modal analysis. One submodule is dedicated to parametric sensitivity studies. These studies are conducted to determine the impact of critical vehicle design parameters on stability and performance. The parametric sensitivity studies will include aero, propulsion, structural, and control parameters of interest. The Control Analysis and Design module provides templates for design of several classical and modern controllers and associated control-centric trade-off studies.

2) Uncertainty Analysis Module: One of the important sub-modules in Postprocessing Module is the uncertainty analysis. Vehicle design parameter uncertainties are obtained from discipline specific knowledge base. This module contains procedures and supporting software to support mapping of these discipline specific uncertainties through the control system design process to provide best case/worst case scenarios for control system design.

\section{Application of Modeling Tool Suite}

The modeling framework described in this paper was used to generate a suite of six DOF models of a facsimile of NASA's Vision Vehicle configuration at different flight conditions. Given below are the flight conditions at a point on a descent trajectory. The flight is un-powered (gliding) flight after engine is turned off. The flight conditions are: Altitude: 95, $100 \mathrm{ft}$, Mach: 4.27, Flight path angle: -38.2 deg, and Velocity: $4219.4 \mathrm{ft} / \mathrm{sec}$. The geometrical and inertial parameters of the vehicle are: Length: $150 \mathrm{ft}$, C.G. location: $950 \mathrm{ft}$ from nose, Span: $75 \mathrm{ft}$, Mass: 285, $459 \mathrm{lbs}$, Non-zero moment of inertias: $I_{x x}: 723,943$ slug- $\mathrm{ft}^{2}, I_{x z}:-72,394$ slug- $\mathrm{ft}^{2}, I_{y y}: 4,959,696$ slug- $\mathrm{ft}^{2}, I_{z z}: 4,304,950$ slug- $\mathrm{ft}^{2}$. The aerodynamic forces acting on the vehicle are: $F_{x}$ : $-37,055 \mathrm{lbs}, F_{y} \sim 0 \mathrm{lbs}, F_{z}=-279,007 \mathrm{lbs}$. The elevator deflection needed to trim the vehicle is $14.5 \mathrm{deg}$.

The structural flexibility for this case is modeled using beam approximation. The flexibility is modeled using two bending modes - one longitudinal (in $x z$ plane) and one lateral (in $x y$ plane). The modal solutions for the beam equations were solved for the free-free boundary condition yielding the following expressions for mode shapes and modal frequencies.

$$
\Phi_{\tau}(x)=\cos \left(\frac{n \pi x}{l}\right) ; \quad \omega_{\tau n}=\frac{n p i}{l} \sqrt{\frac{(G J 2 \pi)}{\left(\rho I_{p}\right)}}
$$

$\Phi_{\tau}(x)$ is the $n^{t h}$ eigenmode corresponding $n^{\text {th }}$ eigenfrequency $\omega_{\tau n}$. In the above relations $l$ is the vehicle length, $G J$ is the Saint Venant torsional rigidity, $\rho$ is the mass density and $I_{p}$ is the polar moment of inertia. Similarly for the bending dynamics in both the $x z$ and $x y$ planes we have:

$$
\begin{aligned}
\Phi_{x z} & =\sin \left(\zeta_{n} x\right)+\sinh \left(\zeta_{n} x\right)+\beta_{n}\left(\cos \left(\zeta_{n} x\right)+\cosh \left(\zeta_{n} x\right)\right) \\
\beta_{n} & =\frac{\left(\cos \left(\zeta_{n} l\right)-\cosh \left(\zeta_{n} l\right)\right)}{\left(\sin \left(\zeta_{n} l\right)+\sinh \left(\zeta_{n} l\right)\right)} ; \quad \omega_{x z n}=\zeta_{n}^{2} \sqrt{\frac{E I 2 \pi}{m l}}
\end{aligned}
$$

In these equations $m$ is the mass per unit length, $E$ is the elastic modulus $I$ is the area moment of inertia for the cross section about the $z$ or $y$ axis depending on the direction of bending (in this case $z$ ). After mass normalizing and mapping these modal deformations to the vehicle geometry various mode shapes are obtained. For the example flight condition selected above, the first bending mode in each direction longitudinal and lateral (see Fig. 4)- is selected for modeling.
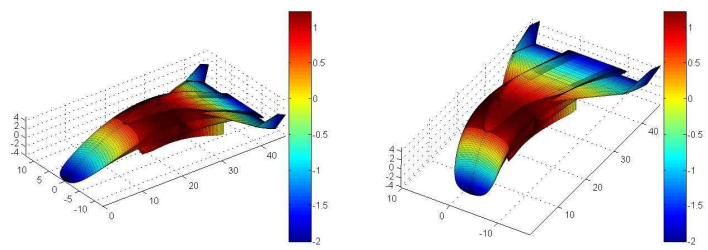

Fig. 4. First longitudinal and lateral bending modeshape

The state space model of the vehicle at this selected flight condition is given by:

$$
\dot{x}(t)=A x(t)+B u(t)
$$

where, the state vector is given by:

$x=\left[h, u, \alpha, \theta, q, \beta, \phi, \psi, r, p, \eta_{1}, \dot{\eta}_{1}, \eta_{2}, \dot{\eta}_{2},\right]$. where $\eta$ 's are the modal coordinates associated with two bending modes. The system matrices are given by:

$$
A(1: 14,1: 7)=
$$

$\left(\begin{array}{ccc}0 & 0 & -4164.8 \\ 0 & 0.001 & -15.66 \\ 0 & 0.0 & -0.065 \\ 0 & 0 & 0 \\ 0 & 0.0 & 3.61 \\ 0 & 0 & 0 \\ 0 & 0 & 0 \\ 0 & 0 & 0 \\ 0 & 0 & 0 \\ 0 & 0 & 0 \\ 0 & 0 & 0 \\ 0 & 0 & 0 \\ 0 & 0 & 0 \\ 0 & 0 & 0\end{array}\right.$
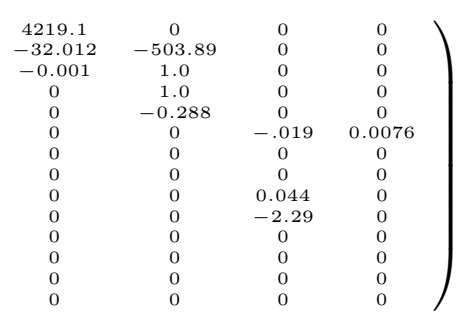

$A(1: 14,8: 14)=$

$\left(\begin{array}{ccc}0 & 0 & 0 \\ 0 & 0 & 0 \\ 0 & 0 & 0 \\ 0 & 0 & 0 \\ 0 & 0 & 0 \\ 0 & -1.0 & 0.120 \\ 0 & 0.1085 & 1.006 \\ 0 & 1.006 & 0 \\ 0 & -0.0449 & 0.015 \\ 0 & 0.0785 & -0.2078 \\ 0 & 0 & 0 \\ 0 & 0 & 0 \\ 0 & 0 & 0 \\ 0 & 0 & 0\end{array}\right.$
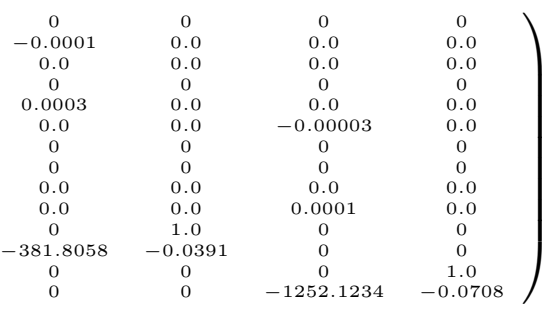

$B=\left(\begin{array}{cc}0 & 0 \\ -3.40 & -3.40 \\ -0.002 & -0.002 \\ 00 & 0 \\ -0.785 & -0.785 \\ 0.001 & -0.001 \\ 00 & 0 \\ 0 & 0 \\ -0.516 & 0.516 \\ 2.42 & -2.42 \\ 0 & 0 \\ -403.23 & -403.23 \\ 0 & 0 \\ -117.90 & 117.90\end{array}\right.$

0
-0.28
-0.001
0
-0.3414
0.0
0
0
-0.059
1.29
0
-185.7
0
-07.75

0

$\begin{array}{cc}0 & 0 \\ -0.28 & -0.01\end{array}$

$\begin{array}{lll}-0.28 & -0.01 & 0 \\ -0.001 & 0.0 & 0.0\end{array}$

$\begin{array}{ccc}-0.001 & 0.0 & 0.0 \\ 0 & 0 & \end{array}$

$\begin{array}{ccc}0 & 0 & \\ -0.3414 & -0.006 & 0.006\end{array}$

$\begin{array}{ccc}0.0 & 0.00 & 0.00\end{array}$

0.0

$\begin{array}{ll}0 & 0 \\ 0 & 0 \\ 0 & -0.002\end{array}$

$\begin{array}{ccc}0.059 & -0.002 & -0.002\end{array}$

$\begin{array}{ccc}-1.29 & 0.028 & 0.028\end{array}$

0

-185.7
0

0

-3.91
0

$\left.\begin{array}{cc}0 & 0.91 \\ -0.72 & -0.72\end{array}\right)$

The input $u(t)$ consists of control surface deflections. 
$u(t)=\left[\delta_{L E}, \delta_{R E}, \delta_{L A}, \delta_{R A}, \delta_{L R}, \delta_{R R}\right]^{T}$, where subscripts $L E$ and $R E$ denote left and right elevators, $L A$ and $R A$ denote left and right ailerons, and $L R$ and $R R$ denote left and right rudder. Since this is unpowered flight condition there is no control input due to cowl flap and fuel flow rate.

The longitudinal and lateral mode characteristics for the linearized model at the above mentioned flight condition are given in Table I.

TABLE I

LONGITUDINAL AND LATERAL DYNAMICS

\begin{tabular}{|c|c|}
\hline Eigenvalue & Mode \\
\hline \multicolumn{2}{|c|}{ Longitudinal } \\
\hline $0.00 \mathrm{E}+00$ & Altitude \\
\hline$-2.50 \mathrm{E}+00$ & Short period \\
\hline $2.21 \mathrm{E}+00$ & Short period \\
\hline $1.16 \mathrm{E}-02$ & Phugoid \\
\hline$-6.95 \mathrm{E}-03$ & Phugoid \\
\hline $\begin{array}{l}-1.95 \mathrm{e}-002+1.95 \mathrm{e}+001 \mathrm{i} \\
-1.95 \mathrm{e}-002-1.95 \mathrm{e}+001 \mathrm{i}\end{array}$ & Longitudinal Bending \\
\hline \multicolumn{2}{|c|}{ Lateral } \\
\hline $0.00 \mathrm{e}+000$ & Heading instability \\
\hline $5.54 \mathrm{e}-001$ & Rolling convergence \\
\hline $1.29 \mathrm{e}-002$ & Spiral like \\
\hline $\begin{array}{l}-4.11 \mathrm{e}-001+2.15 \mathrm{e}-001 \mathrm{i} \\
-4.11 \mathrm{e}-001-2.15 \mathrm{e}-001 \mathrm{i}\end{array}$ & Dutch roll \\
\hline $\begin{array}{l}-3.54 \mathrm{e}-002+3.54 \mathrm{e}+001 \mathrm{i} \\
-3.54 \mathrm{e}-002-3.54 \mathrm{e}+001 \mathrm{i}\end{array}$ & Lateral bending \\
\hline
\end{tabular}

\section{A. Trade studies}

The modeling framework presented in this paper was used to generate suite of models at different flight conditions on the trajectory to assess stability and performance at various points along trajectory and determine associated control requirements. In addition, several trade-off studies were also conducted for various parametric perturbations. One good example of trade studies needed at early stages in the vehicle design is the sizing of control surfaces. For the vehicle considered in this paper a study was conducted for determining the elevator sizing for different combinations of C.G. locations and elevator deflections required to trim and balance the vehicle. The result from such analysis is given in Fig. 5. From this plot one can determine what size of elevator is needed for a desired range of elevator deflections and different C.G. locations. There are numerous such analyses conducted for the concept vehicle modeled in this paper, however, due to space limitations all the results could not be included. Interested readers can contact authors to obtain different models developed by authors for their own control design analysis and synthesis.

\section{CONCLUding REMARKS}

There exists large body of literature in the disciplinespecific areas in scramjet-powered hypersonic vehicles; however, none of the existing tools lends themselves readily to help formulate/develop control-centric dynamic models of such vehicles having desired order and fidelity. The work described in this paper was aimed at developing a suite of

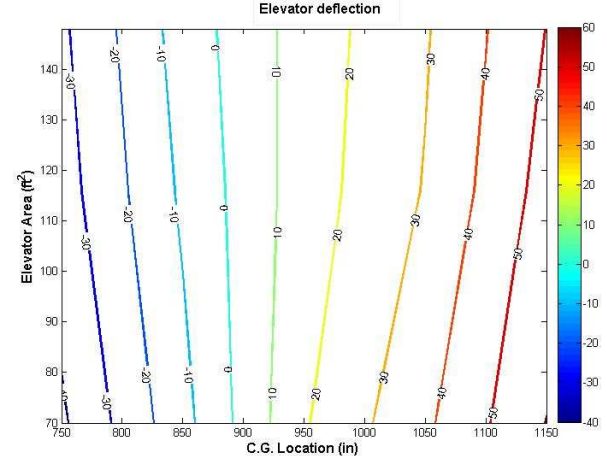

Fig. 5. Control surface deflection required to achieve trim and balance

multidisciplinary modeling tools which integrate disciplinary states-of-the-art to enable timely development of dynamic models of concept vehicles with varying fidelity and plant size. These tools will facilitate control-relevant analysis and design during early stages of the vehicle design process to maximize overall vehicle stability and performance.

\section{ACKNOWLEDGMENTS}

The authors gratefully acknowledge NASA's support through NRA Award \# NNL08AA38C.

\section{REFERENCES}

[1] J. Pittman and P. A. Bartolotta, "Fundamental Aeronautics Hypersonics Project Reference Document,". Technical Report, National Aeronautics and Space Administration (NASA), 2006.

[2] D.K. Schmidt, "Dynamics and Control of Hypersonic Aeropropulsive/Aeroelastic Vehicles,"AIAA Guidance, Navigation and Control Conference, Paper No. 1992-4326, 1992.

[3] F. R. Chavez and D. K. Schmidt, "Analytical Aeropropulsive/Aeroelastic Hypersonic-Vehicle Model with Dynamic Analysis," J. of Guidance, Control and Dynamics, pp. 1308 - 1319, 1994.

[4] M. A. Bolender and D. B. Doman, "A Non-Linear Longitudinal Dynamical Model of an Air-Breathing Hypersonic Vehicle,", J. of Spacecraft and Rockets, Vol. 44, No. 2, pp. 373 - 3872007.

[5] A. Clark, C. Wu, M. Mirmirani, S. Choi and M. Kuipers, "Development of an Airframe-Propulsion Integrated Hypersonic Vehicle Model," $44^{\text {th }}$ AIAA Aerospace Sciences Meeting and Exhibit, Paper No. 2006-218, 2006.

[6] M. Oppenheimer and D. Doman, "A Hypersonic Vehicle Model Developed with Piston Theory," Technical Report, AFRL, Wright Patterson Air Force Base, pp. 1 - 26, 2006.

[7] J.J. McNamara and P.P. Friedmann, "Aeroelastic and Aerothermoelastic Analysis of Hypersonic Vehicles: Current Status and Future Trends," $14^{\text {th }}$ AIAA/AHI Space Planes and Hypersonic Systems and Technologies Conference,Paper No. 2006-8058, Canberra, AUS, November 6-9, 2006.

[8] Sean M. Torrez, James F. Driscoll, Michael A. Bolender, Michael W. Oppenheimer, David B. Doman, Effects of Improved Propulsion Modelling on the Flight Dynamics of Hypersonic Vehicles, AIAA Atmospheric Flight Mechanics Conference and Exhibit, Honolulu, Hawaii, Paper \# AIAA 2008-6386, August 18-21, 2008.

[9] S. M. Torrez, N. A. Scholten, D. J. Micka, J. F Driscoll, M. A. Bolender, D. B. Doman, M. W. Oppenheimer, A Scramjet Engine Model Including Effects of Precombustion Shocks and Dissociation, 44th AIAA/ASME/SAE/ASEE Joint Propulsion Conference \& Exhibit, Hartford, CT. Paper \# AIAA 2008-4619, July 21-23, 2008.

[10] v. E. Rudd, L. and D. Pines, Integrated Propulsion Effects on the Dynamic Stability and Control of Hypersonic Waveriders, AIAA 2000 3826, 2000 . 